\title{
Estudio acústico de las consonantes de la lengua yagua
}

\section{An Acoustic Study of Yagua Consonants}

\section{Luis Carlos Peláez Torres}

Universidad Nacional Mayor de San Marcos, Lima, Perú

Contacto: luis.pelaez@unmsm.edu.pe

https://orcid.org/ 0000-0002-6197-4015

\section{RESUMEN}

Esta investigación presenta una descripción acústica de los sonidos consonánticos del yagua, una lengua aislada perteneciente a la familia pebayagua y que se habla en la región nororiental del Perú. El número de personas que componen la sociedad yagua actual asciende a aproximadamente 5679. La población se distribuye principalmente en las provincias de Mariscal Ramón Castilla, Putumayo y Maynas, en el departamento de Loreto, principalmente en las zonas fronterizas con Colombia y Brasil. El objetivo de este estudio es establecer el número de consonantes de la lengua yagua y detallar sus propiedades acústicas. Sobre la base de la fonética acústica y tras un acuerdo con los colaboradores yagua a fin de realizarles entrevistas para obtener estos datos necesarios para la investigación, se elicitó un conjunto de 200 palabras a 9 colaboradores de las comunidades del río Amazonas. Posteriormente, se realizó el procesamiento de las grabaciones a través del programa informático Praat, que permitió reconocer las características acústicas de los sonidos de esta lengua. Entre los resultados más resaltantes, se ha podido determinar la existencia de 20 sonidos consonánticos en yagua: $[\mathrm{p}],\left[\mathrm{p}^{\mathrm{w}}\right]$, $\left.[\mathrm{t}],[\mathrm{k}],[\mathrm{m}],\left[\mathrm{m}^{\mathrm{w}}\right],[\mathrm{mb}],[\mathrm{n}],[\mathrm{n}],\left[{ }^{\mathrm{n}} \mathrm{d}\right],[\mathrm{t}]\right],[\mathrm{s}],\left[\int\right],[\mathrm{r}]$, $[r],[r],[w],[\beta],[j]$ y $[h]$.

Palabras clave: Fonética acústica; Sonidos consonánticos; Lengua yagua; Praat; Río Amazonas.

\begin{abstract}
This research presents an acoustic description of the consonant sounds of the yagua language, an isolated language belonging to the Peba-yagua family which is spoken in the northeastern region of Peru. The number of people in the current yagua society amounts to approximately 5,679. The population is distributed mainly in the provinces of Mariscal Ramón Castilla, Putumayo, and Maynas in the department of Loreto, mainly in the border areas with Colombia and Brazil. The objective of this study was to establish the number of consonants in the Yagua language and detail their acoustic properties. On the basis of acoustic phonetics and after an agreement with the Yagua collaborators to carry out interviews to them in order to obtain this data for the investigation, a set of 200 words was elicited from 9 speakers from the Amazon River communities. Subsequently, the recordings were processed using the Praat computer program, which made it possible to recognize the acoustic characteristics of the sounds of the Yagua language. Among the most noteworthy results, it has been possible to determine the existence of 20 consonant sounds in the Yagua language: $[\mathrm{p}],\left[\mathrm{p}^{\mathrm{w}}\right]$, $[\mathrm{t}],[\mathrm{k}],[\mathrm{m}],\left[\mathrm{m}^{\mathrm{w}}\right],\left[{ }^{\mathrm{m} b}\right],[\mathrm{n}],[\mathrm{n}],\left[{ }^{\mathrm{n}} \mathrm{d}\right],[\mathrm{t} J],[\mathrm{s}],\left[\int\right],[\mathrm{r}]$, $[\mathrm{c}],[\mathrm{c}],[\mathrm{w}],[\mathrm{\beta}],[\mathrm{j}]$ and $[\mathrm{h}]$.
\end{abstract}

Keywords: Acoustic Phonetics; Consonant Sounds; Yagua Language; Praat; Amazon River. 


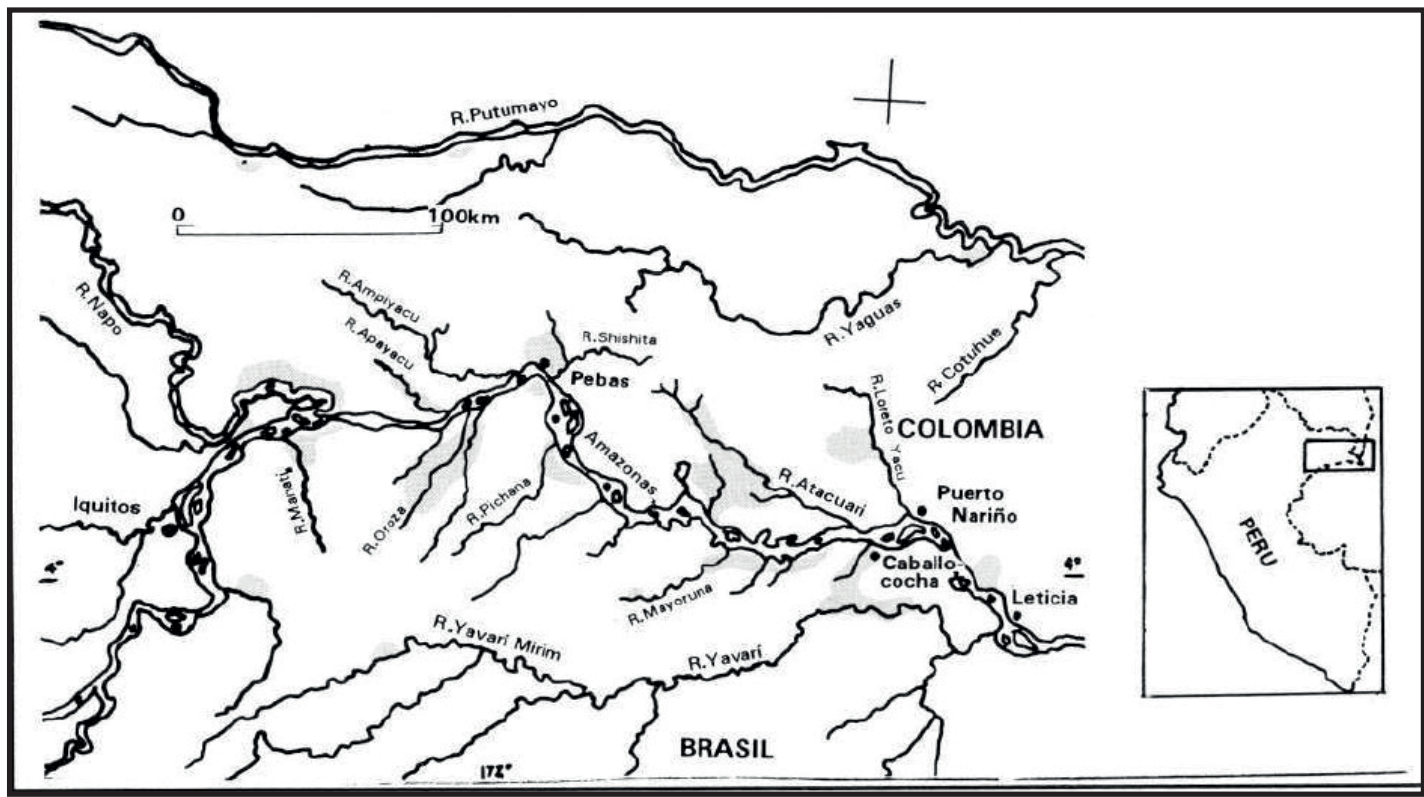

Figura 1. Provincias de Ramón Castilla, Maynas y Putumayo, así como la zona de dispersión en la que se encuentra el pueblo yagua. Fuente: Chaumeil, 1994, p. 154.

\section{Introducción}

El pueblo yagua se halla disperso por diversos puntos geográficos dentro de las provincias de Mariscal Ramón Castilla, Maynas y Putumayo, todas en el departamento de Loreto. Su espacio territorial actual aes producto de una serie de traslados generados por agentes externos que fragmentaron su territorio tradicional a lo largo de la historia poscolonial del Perú (Chaumeil, 1994). En la figura 1 se muestran las áreas en las que habita el pueblo yagua en la actualidad.

La lengua yagua pertenece a la familia lingüística peba-yagua, que comprende las lenguas peba, yagua y yameo (Ministerio de Educación, 2013). En la actualidad, el yagua es la única que subsiste de estas tres y es considerada una lengua aislada (Peña, 2009; Chaumeil, 1987). En cuanto a la población yagua, según indica el Documento Nacional de Lenguas Originarias del Perú (Ministerio de Educación, 2013, p. 63), existe un total de 5679 pobladores. No obstante, no está comprobado que todos ellos sean hablantes de la lengua ni que existan hablantes monolingües. Sobre los estudios lingüísticos del yagua, se destacan, principalmente, investigaciones sobre sintaxis, como las de Doris Payne (1985). No obstante, en cuanto a fonética y fonología, aunque la referida autora explica determinados aspectos de algunos fenómenos y reglas fonológicas, se limita a describir un inventario propuesto por ella misma. Sobre el inventario consonántico del yagua, Payne (1985, p. 30) indica que existe un total de 20 sonidos consonánticos en esta lengua, los cuales se manifiestan a partir de 11 fonemas. En tal sentido, la citada autora presenta una tabla de dichos sonidos y su distribución alofónica ${ }^{1}$.

Cuadro 1. Fonemas y alófonos de las consonantes del yagua según Payne (1985)

\begin{tabular}{|c|c|c|c|c|c|}
\hline & Labiales & Alveolares & Palatales & Velares & Posvelares \\
\hline Oclusivas & $\begin{array}{ll}\mathrm{p} / & {[\mathrm{p} w]} \\
& {[\mathrm{p}]}\end{array}$ & $\begin{array}{ll}/ \mathrm{t} / & {[\mathrm{t}]} \\
& {[\mathrm{c}]}\end{array}$ & & $/ \mathrm{k} / \quad[\mathrm{k}]$ & \\
\hline Nasales & $\begin{array}{cc} & {\left[\mathrm{m}^{\mathrm{w}}\right]} \\
& \mathrm{m} / \\
& {[\mathrm{m}]} \\
& {\left[\mathrm{m}^{\mathrm{b}}\right]}\end{array}$ & $\begin{array}{ll} & {[\mathrm{n}]} \\
/ \mathrm{n} / & {[\mathrm{n}]} \\
& {\left[\mathrm{n}^{\mathrm{d}}\right]}\end{array}$ & & & \\
\hline Africadas & & & $/ \overline{\mathrm{t} t}[\mathrm{t}] \mathrm{t}]$ & & \\
\hline Fricativas & & $\begin{array}{cc}/ \mathrm{s} / & {[\widehat{\mathrm{ts}}]} \\
& {[\hat{\mathrm{t}} \mathrm{s}] \sim[\mathrm{f}]}\end{array}$ & & & \\
\hline Vibrantes & & $/ / \check{r} / \quad[\check{r}]$ & & & \\
\hline Aproximantes & $\begin{array}{ll} & {[\mathrm{w}]} \\
& {[\beta]}\end{array}$ & & $/ j / \quad$ [j] & & $/ \mathrm{h} / \quad[\mathrm{h}]$ \\
\hline
\end{tabular}

Fuente: adaptado de Payne, 1985, p. 29.

Sobre la base de esta información, se pudo establecer ejemplos de pares mínimos entre los 11 fonemas consonánticos. $\mathrm{Al}$ respecto, obsérvese las entradas propuestas en el cuadro 2. 
Guadro 2. Ejemplos de oposición fonémica por pares mínimos en yagua

\begin{tabular}{|c|c|c|c|c|c|c|c|c|}
\hline$<\mathrm{p} / \mathrm{m}$ & $t / t 5$ & $k / p$ & $\mathrm{~s} / \mathrm{r}$ & $\mathrm{h} / \mathrm{r}$ & $\mathrm{m} / \mathrm{r}$ & $\mathrm{n} / \mathrm{t} \tau$ & $r / w$ & $y / w$ \\
\hline $\begin{array}{l}\text { hapi } \\
\text { 'totora' }\end{array}$ & $\begin{array}{l}\text { wata } \\
\text { 'mono } \\
\text { blanco' }\end{array}$ & $\begin{array}{l}\text { kot } \pi i \\
\text { 'loro' }\end{array}$ & $\begin{array}{l}\text { wasa } \\
\text { 'seco' }\end{array}$ & $\begin{array}{l}\text { hapa } \\
\text { 'guacamayo' }\end{array}$ & $\begin{array}{l}\text { tama } \\
\text { 'pero' }\end{array}$ & $\begin{array}{l}\text { winu } \\
\text { 'soltero' }\end{array}$ & $\begin{array}{l}\text { kara } \\
\text { ¡caramba!' }\end{array}$ & $\begin{array}{l}\text { yawa } \\
\text { 'yagua' }\end{array}$ \\
\hline $\begin{array}{l}\text { hami } \\
\text { 'grande' }\end{array}$ & $\begin{array}{l}\text { watJa } \\
\text { 'mono } \\
\text { guapo' }\end{array}$ & $\begin{array}{l}\operatorname{pot} \pi i \\
\text { 'hija' }\end{array}$ & $\begin{array}{l}\text { wara } \\
\text { 'callado' }\end{array}$ & $\begin{array}{l}\text { rapa } \\
\text { 'afrecho' }\end{array}$ & $\begin{array}{l}\text { 'tara' } \\
\text { 'cosa' }\end{array}$ & $\begin{array}{l}\text { witjú } \\
\text { 'especie } \\
\text { de ave' }\end{array}$ & $\begin{array}{l}\text { kawa } \\
\text { 'especie de } \\
\text { ave' }\end{array}$ & $\begin{array}{l}\text { rawa } \\
\text { 'veneno' }\end{array}$ \\
\hline
\end{tabular}

Cabe indicar que, a pesar de la importancia que suponen los alcances de Payne para la comprensión del yagua, la descripción fonético-fonológica que presenta no es exhaustiva, pues, en cuanto al aspecto fonológico, la autora no realiza una descripción de la prosodia de la lengua. Sin embargo, Paul Powlison (2008) señala que esta lengua presenta un acento tonal, aunque no realiza una explicación sobre tal fenómeno ni lo describe como tal. En cuanto a lo fonético, es necesario mencionar que las investigaciones de ambos autores carecen de evidencia acústica que confirme o determine los sonidos consonánticos de esta lengua. Por ello, bajo la problemática presentada sobre la no exhaustividad en el estudio de las consonantes en yagua, surge la pregunta: ¿cuál es el inventario de los sonidos consonánticos en la lengua yagua? En tal sentido, el objetivo de esta investigación fue determinar el inventario de sonidos consonánticos de la lengua yagua mediante una descripción acústica de sus principales propiedades fonéticas.

\section{Marco teórico metodológico}

\subsection{Antecedentes}

Payne (1985) expone un inventario de 11 fonemas y 19 fonos consonánticos. Por su parte, Powlison (2008) presenta algunos de los sonidos consonánticos en términos grafémicos y realizando comparaciones con el castellano. Así, no muestra un inventario fonológico de la lengua, sino más bien un alfabeto, con el cual presupone la intuición fonológica propia del lector al que se dirige. Por otro lado, Peña (2009), sobre la base de otros investigadores (Payne, Powlison, Gordon, etc.) ofrece un resumen fonológico de la lengua yagua. En cuanto a las consonantes, señala que existen once fonemas: /p/, /t/, /k/, /m/, /n/, /s/, /t T/, / / /, $/ \mathrm{w} /, \mathrm{j} / \mathrm{y} / \mathrm{h} /$.

\subsection{Bases teóricas}

La fonética acústica admite describir las características de los sonidos del habla en términos numéricos. Esta cuantificación permite medir los valores de los sonidos para poder describirlos en términos acústicos por medio de un espectrograma que, en la presente investigación, se ha obtenido a través del programa informático llamado Praat. Su representación es tridimensional: señala los valores de frecuencia, amplitud y tiempo. Tal como indica Peter Ladefoged, "la imagen [...] es denominada espectrograma. [...]. En los espectrogramas el tiempo corre de izquierda a derecha, la frecuencia de los componentes se muestra en escala vertical y la intensidad de cada uno se muestra en el grado de oscuridad" (Ladefoged y Johnson, 2010, p. 194). Cabe señalar que los parámetros generales para distinguir todas las consonantes que se aplican en esta investigación son la duración del segmento y la barra de sonoridad. El primer indicador nos permitirá comparar los valores temporales de cada una de las clases de consonantes y establecer un posible contraste fonológico dual, es decir, entre consonantes cortas o largas (Ashby, 2011, p. 141). Por su parte, la barra de sonoridad da la posibilidad de distinguir consonantes sordas de las sonoras, pues se manifiesta como una notable concentración de energía en las frecuencias bajas en el caso de las consonantes sonoras (Johnson, 2003, p. 140).

\subsection{Metodología}

Se trata de una investigación descriptiva que enumera y detalla los sonidos consonánticos de la lengua yagua. La técnica de recolección de datos que se empleó fue la elicitación a través de un protocolo de investigación que contiene el listado de 200 palabras de Morris Swadesh (véase el Anexo I), el cual está constituido 
por un vocabulario que se considera esencial a cualquier lengua y que difícilmente podría originarse en préstamos de otra lengua (Aguilar-Porras, 2013, pp. 125-129). Para tal fin, se utilizó una lista de 200 palabras en español, las cuales el colaborador debía traducir al yagua, por indicación oral del entrevistador, una por una en el mismo momento y enunciarlas tres veces. La palabra que se utilizaría como muestra para el análisis acústico sería siempre la producida en el medio de esas tres. A continuación, en el cuadro 3, se presenta el listado completo de las palabras que se elicitaron en las entrevistas, junto con la palabra equivalente propuesta por Powlison (2008) que se tuvo como referencia solo para conocimiento del investigador.

Cuadro 3. Listado de palabras empleadas en la elicitación

\begin{tabular}{|c|c|c|}
\hline & Palabra en español & $\begin{array}{l}\text { Palabra en yagua } \\
\text { (escrita según el alfabeto } \\
\text { de Powlison, 2008) }\end{array}$ \\
\hline 1 & animal & javada \\
\hline 2 & perro & nibi \\
\hline 3 & pájaro & viche \\
\hline 4 & culebra & codiy \\
\hline 5 & pez, pescado & quiva \\
\hline 6 & piojo & runi \\
\hline 7 & lombriz (no gusano) & jntyoda \\
\hline 8 & pasto & pastu \\
\hline 9 & árbol & ninu \\
\hline 10 & nombre & jitya \\
\hline 11 & padre & jay \\
\hline 12 & madre & junoda \\
\hline 13 & esposo & vanu \\
\hline 14 & esposa & rimityoda \\
\hline 15 & hombre & vanu \\
\hline 16 & mujer & vatura \\
\hline 17 & niño & dera \\
\hline 18 & persona & nijyami \\
\hline 19 & cabeza & juno \\
\hline 20 & oreja & tuvay \\
\hline 21 & ojo & nisiy \\
\hline 22 & nariz & nuru \\
\hline 23 & boca & juto \\
\hline
\end{tabular}

\begin{tabular}{|c|c|c|}
\hline 24 & lengua & dachiy \\
\hline 25 & diente & janu \\
\hline 26 & cuello & tunuvu \\
\hline 27 & barriga & pude \\
\hline 28 & espalda & runu \\
\hline 29 & rabo, cola & musiy \\
\hline 30 & pierna & duse \\
\hline 31 & pie & numutu \\
\hline 32 & ala & nipya \\
\hline 33 & mano & jomutu \\
\hline 34 & corazón & jachiy \\
\hline 35 & tripas, intestinos & siquidi \\
\hline 36 & hígado & juvatu \\
\hline 37 & hueso & $d u$ \\
\hline 38 & carne & saviy \\
\hline 39 & grasa & nidi \\
\hline 40 & piel & jay \\
\hline 41 & cabello (o pelo) & nojasiy \\
\hline 42 & pluma & $\mathrm{mu}$ \\
\hline 43 & sangre & ndaja \\
\hline 44 & raíz & jntiy \\
\hline 45 & corteza & jay \\
\hline 46 & hoja & navi \\
\hline 47 & flor & ninubi \\
\hline 48 & fruta & dabi \\
\hline 49 & semilla & jatadi \\
\hline 50 & palo & jipu \\
\hline 51 & ceniza & pupujuru \\
\hline 52 & montaña & musiy \\
\hline 53 & bosque & curuju \\
\hline 54 & río & $\mathrm{mu}$ \\
\hline 55 & lago & jmityo \\
\hline 56 & mar & nava \\
\hline 57 & agua & ja \\
\hline 58 & hielo & sudochiy \\
\hline 59 & fuego & jiday \\
\hline 60 & humo & navachiy \\
\hline 61 & tierra & mucadi \\
\hline 62 & polvo & jiru \\
\hline 63 & arena & ticha \\
\hline
\end{tabular}




\begin{tabular}{|c|c|c|c|c|c|}
\hline 64 & piedra & avichu & 104 & caminar, andar & rupi \\
\hline 65 & carretera (camino, trocha) & un & 105 & volar & ratye \\
\hline 66 & huevo & vada & 106 & nadar & nuniy \\
\hline 67 & Iluvia & rumura & 107 & voltear, girar & daputa \\
\hline 68 & nieve & jarcha & 108 & jugar & tisa \\
\hline 69 & neblina & ajeravurya & 109 & ver & junuy \\
\hline 70 & cielo & jarichu & 110 & oler & nari \\
\hline 71 & nube & nuvachiy & 111 & oír & tuvachu \\
\hline 72 & viento & richo & 112 & saber & datya \\
\hline 73 & sol & jñi & 113 & pensar & jachipiya \\
\hline 74 & estrella & napay & 114 & temer & sabuy \\
\hline 75 & día & narya & 115 & contar & saranu \\
\hline 76 & noche & nupora & 116 & decir & jutay \\
\hline 77 & año & nijya & 117 & cantar & muyivay \\
\hline 78 & cuerda & judiju & 118 & reír & todi \\
\hline 79 & sal & tivyiy & 119 & comer & jimiy \\
\hline 80 & yo & ray & 120 & beber, tomar & jatu \\
\hline 81 & tú, usted & $\mathrm{ji}$ & 121 & chupar & rupay \\
\hline 82 & él & ni & 122 & morder & sado \\
\hline 83 & ellos & riy & 123 & escupir & jtochiy \\
\hline 84 & nosotros & nuy & 124 & vomitar & jiyaniy \\
\hline 85 & ustedes, vosotros & jirye & 125 & rascar & amani \\
\hline 86 & ¿quién? & chi & 126 & cazar & tanchu \\
\hline 87 & ¿qué? & tara & 127 & quemar & rupay \\
\hline 88 & este & jiñi mutijo & 128 & coser & siniy \\
\hline 89 & ese, aquel & dañu, jiryamuy & 129 & atar, amarrar & puchu \\
\hline 90 & vivir & vicha & 130 & jalar & ramichu \\
\hline 91 & morir & ditya & 131 & tirar, lanzar & jupatya \\
\hline 92 & congelar, helarse & - & 132 & empujar & jupa \\
\hline 93 & hinchar & pochu & 133 & apretar & naya \\
\hline 94 & caer-(se) & juy & 134 & cavar, escarbar & jumiy \\
\hline 95 & respirar & narye & 135 & lavar & suta \\
\hline 96 & soplar & duye & 136 & limpiar, enjugar & puryi \\
\hline 97 & dormir & may & 137 & sobar, frotar & sityaniya \\
\hline 98 & echar-(se) & jatya & 138 & dar & say \\
\hline 99 & sentar-(se) & masa & 139 & agarrar, sostener & ryi \\
\hline 100 & parar-(se) & raniy & 140 & cortar & jaruy \\
\hline 101 & flotar, rebalsar & pupaya & 141 & partir & ruve \\
\hline 102 & fluir & si & 142 & pelear, luchar & juvatyi \\
\hline 103 & venir & juniy & 143 & golpear & juvay \\
\hline
\end{tabular}




\begin{tabular}{|c|c|c|}
\hline 144 & penetrar (perforando) & ramujetya \\
\hline 145 & matar & juvay \\
\hline 146 & liso & judutyasi \\
\hline 147 & caliente & vanuqui \\
\hline 148 & frío & canuvu \\
\hline 149 & filudo & jimyura \\
\hline 150 & desafilado & motadi \\
\hline 151 & podrido & purya \\
\hline 152 & recto, derecho & vase \\
\hline 153 & sucio & $\mathrm{mi}$ \\
\hline 154 & pesado & jinra \\
\hline 155 & mojado & japadi \\
\hline 156 & seco & vasa- \\
\hline 157 & negro & vadacu \\
\hline 158 & blanco & pupa- \\
\hline 159 & rojo & runay \\
\hline 160 & amarillo & vasunudi \\
\hline 161 & verde & vasununay \\
\hline 162 & nuevo & vadi- \\
\hline 163 & viejo & tarijevyu \\
\hline 164 & bueno & samuy \\
\hline 165 & malo & nicyaray \\
\hline 166 & aquí, acá & jiyu \\
\hline 167 & ahí, allí, allá & jasiy, muy \\
\hline 168 & cerca & nuji \\
\hline 169 & lejos & ripa \\
\hline 170 & derecha & jntityuday \\
\hline 171 & izquierda & miday \\
\hline 172 & grande & jamura \\
\hline 173 & ancho & jamu- \\
\hline 174 & grueso & jamu- \\
\hline 175 & pequeño & pasi- \\
\hline 176 & delgado & paniyo- \\
\hline 177 & angosto, estrecho & pasi- \\
\hline 178 & largo & jamuca- \\
\hline 179 & corto, bajo & japu- \\
\hline 180 & uno & taraqui \\
\hline 181 & dos & adajuy \\
\hline 182 & tres & mumuri \\
\hline 183 & cuatro & nañujuyu \\
\hline
\end{tabular}

\begin{tabular}{|l|l|l|}
\hline 184 & cinco & tadajyo \\
\hline 185 & muchos & raju \\
\hline 186 & pocos & pasi- \\
\hline 187 & todos & tita \\
\hline 188 & algunos & tavay \\
\hline 189 & otro & ti \\
\hline 190 & donde & jimu \\
\hline 191 & cuándo & nutyerivye \\
\hline 192 & cómo & nutiy \\
\hline 193 & en, adentro & jimu \\
\hline 194 & correcto & vase- \\
\hline 195 & no & ne \\
\hline 196 & y & jarye \\
\hline 197 & porque & daryaju \\
\hline 198 & si & tiy \\
\hline 199 & en & jimu \\
\hline 200 & con & \\
\hline
\end{tabular}

alfabeto de Powlison, 2008)

Cabe señalar que todo el procedimiento se llevó a cabo de forma directa y con el consentimiento expreso de los 9 colaboradores yagua que se ofrecieron para participar en las entrevistas, todos ellos provenientes de diversas comunidades de la cuenca del río Amazonas en el departamento de Loreto. A continuación, se presenta un cuadro con los datos de cada uno de ellos y la codificación empleada para los datos del análisis.

Cuadro 4. Lista de colaboradores y su codificación

\begin{tabular}{|l|l|l|l|}
\hline Colaborador & Género & Lugar de residencia & Código \\
\hline Colaborador 1 & Varón & Atacuari & CC-V-A \\
\hline Colaborador 2 & Varón & Caballococha & AG-V-C \\
\hline Colaborador 3 & Varón & Río Napo & MS-V-R \\
\hline Colaborador 4 & Varón & Putumayo & FP-H-P \\
\hline Colaborador 5 & Varón & Putumayo & HQ-V-P \\
\hline Colaborador 6 & Mujer & Urco Miraño & MM-M-U \\
\hline Colaborador 7 & Varón & Urco Miraño & A-V-U \\
\hline Colaborador 8 & Varón & Urco Miraño & CC-V-U \\
\hline Colaborador 9 & Varón & Santa Rita & WR-V-S \\
\hline
\end{tabular}


Para grabar, se utilizó una grabadora digital Tascam DR-40, un micrófono Shure WH-20 y un cuaderno de apuntes. De esa manera, se realizó la grabación a los colaboradores teniendo como guía el instrumento de recolección de datos (Anexo I). Una vez obtenidos los datos, se segmentaron los audios para luego seleccionar los sonidos consonánticos que sirvan de muestra para evaluar las particularidades que se buscan. Esta labor se realizó a través del programa informático Praat, versión 6.1.15, con el cual se pudo extraer los espectrogramas de cada sonido. Finalmente, se clasificaron y describieron las características acústicas de las consonantes en función de las correspondencias fonológicas descritas en los antecedentes.

\section{Resultados}

\subsection{Consonantes oclusivas}

La lengua yagua cuenta con tres sonidos oclusivos sordos: bilabial [p], alveolar [t] y velar [k]. El rasgo común de estas consonantes es que todas presentan una fase de silencio que corresponde al momento del cierre del punto de articulación, seguida de la barra de explosión y el Voice Onset Time (VOT). Asimismo, al ser sonidos sordos, carecen de la característica barra de sonoridad de las oclusivas sonoras. Véase, a continuación, los espectrogramas de las figuras 2, 3 y 4, en las que se puede observar la constitución acústica de cada una de estas consonantes.

Figura 2. Espectrograma de la palabra ['pø.pø] 'ceniza'

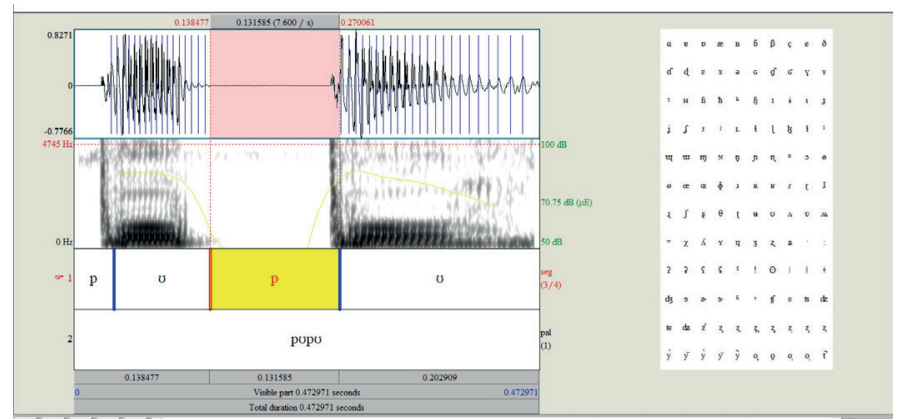

Como se puede ver en cada una de las imágenes, las tres oclusivas sordas guardan un aspecto muy similar. Por ello, es necesario diferenciarlas a través de sus valores de duración de VOT. En el cuadro 5, se muestra la duración en promedio de la fase
Figura 3. Espectrograma de la palabra [sa.nu.mo.'to] 'pie'

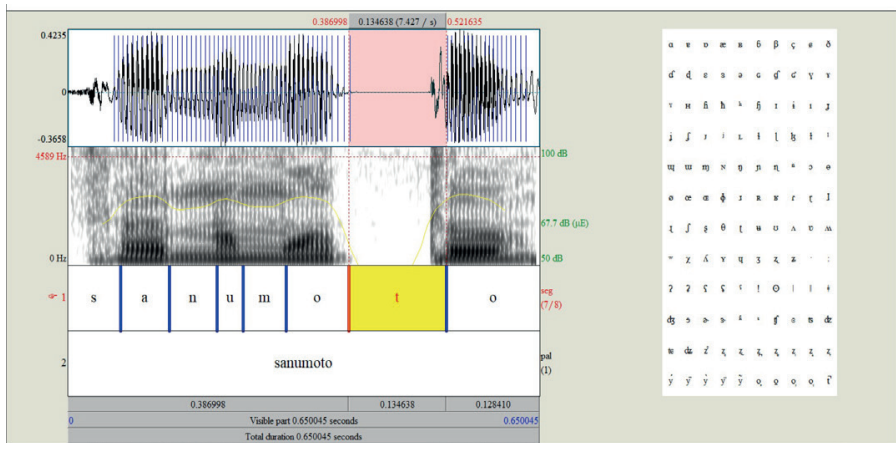

Figura 4. Espectrograma de la palabra [mu.'ko] 'tierra'

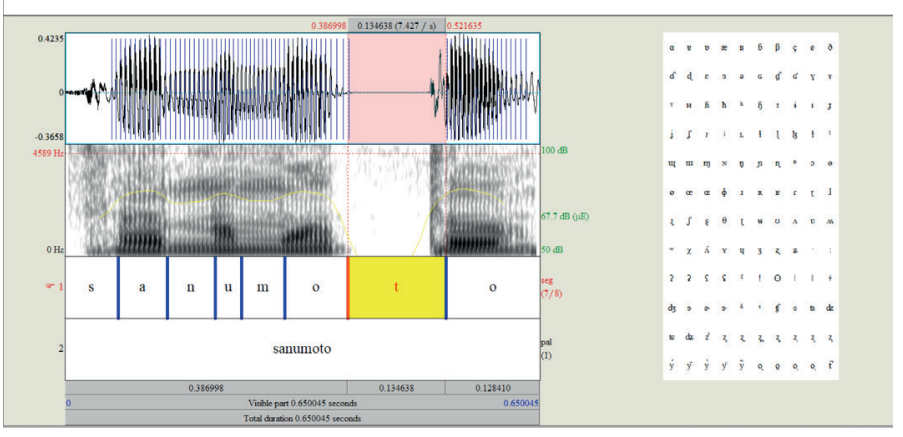

cerrazón, que corresponde acústicamente al silencio y se indica, entre paréntesis y en superíndice, la cifra de la desviación estándar del conjunto de muestras.

A continuación, se muestra la duración del VOT de cada sonido. Cabe indicar que todas las muestras fueron tomadas en contextos semejantes: flanqueados por sonidos vocálicos.

Cuadro 5. Duración de la fase de cierre de las oclusivas sordas

\begin{tabular}{|l|l|c|}
\hline Consonante & Muestras & Duración (seg.) (desv. est.) \\
\hline$[\mathrm{p}]$ & 20 & $0.121(0.018)$ \\
\hline$[\mathrm{t}]$ & 30 & $0.112(0.026)$ \\
\hline$[\mathrm{k}]$ & 25 & $0.093(0.018)$ \\
\hline
\end{tabular}

Guadro 6. Duración del VOT de las oclusivas sordas

\begin{tabular}{|l|l|c|}
\hline Consonante & Muestras & Duración (seg.) (desv. est.) \\
\hline$[p] 20$ & 0.011 & $(0.003)$ \\
\hline$[t] 30$ & 0.023 & $(0.003)$ \\
\hline$[k] 25$ & 0.033 & $(0.004)$ \\
\hline
\end{tabular}


Guadro 7. Promedio de intensidad y duración total de las consonantes oclusivas sordas del yagua

\begin{tabular}{|l|l|l|l|l|l|}
\hline Consonante & Muestras & $\begin{array}{l}\text { Intensidad } \\
(\mathrm{dB}) \text { (desv. est.) }\end{array}$ & $\begin{array}{l}\text { Duración de la } \\
\text { fase de cierre } \\
\text { (seg.) (desv. est.) }\end{array}$ & $\begin{array}{l}\text { Duración del } \\
\text { VOT } \\
\text { (seg.) (desv. est.) }\end{array}$ & $\begin{array}{l}\text { Duración total } \\
\text { (seg.) (desv. est.) }\end{array}$ \\
\hline$[\mathrm{p}]$ & 20 & $71.749(1.546)$ & $0.121(0.018)$ & $0.011(0.003)$ & $0.132(0.019)$ \\
\hline$[\mathrm{t}]$ & 30 & $67.710(1.335)$ & $0.112(0.026)$ & $0.023(0.003)$ & $0.135(0.025)$ \\
\hline$[\mathrm{k}]$ & 25 & $63.391(1.246)$ & $0.093(0.018)$ & $0.033(0.004)$ & $0.126(0.020)$ \\
\hline
\end{tabular}

Como puede apreciarse en los espectrogramas, la consonante con el mayor tiempo de duración de VOT es [k] seguida por [t] y [p]. Cabe indicar que esta progresión es compartida por diversas lenguas del mundo (Jiménez, 2018, p. 127). La suma de los dos valores mencionados anteriormente constituiría el valor total de cada consonante, el que se resume en el cuadro 7 .

\subsection{Consonantes nasales}

La lengua yagua cuenta con tres sonidos nasales: bilabial [m], alveolar [n] y palatal [n]. Estas consonantes presentan una alta concentración de energía en las frecuencias inferiores a los $1000 \mathrm{~Hz}$, a la vez que un debilitamiento de las frecuencias altas, lo que se traduce en la intermitencia que se puede visualizar como manchas blancas en el espectrograma (murmullo nasal). Estas manchas son conocidas como antiformantes y pueden observarse a partir de los 1000 $\mathrm{Hz}$ en frecuencias más altas. Las tres consonantes presentan un FN entre 250 y $350 \mathrm{~Hz}$, característico de estas consonantes. La bilabial [m] y la alveolar [n] presentan un antiformante inferior (AF1) inferior a los $2000 \mathrm{~Hz}$, mientras que la palatal [n] supera a este valor. Al respecto, véanse las figuras 5, 6 y 7, en las que los valores de los formantes nasales se han tomado en el punto medio del segmento.

Figura 5. Espectrograma de la palabra [e.'he.na] 'diente'

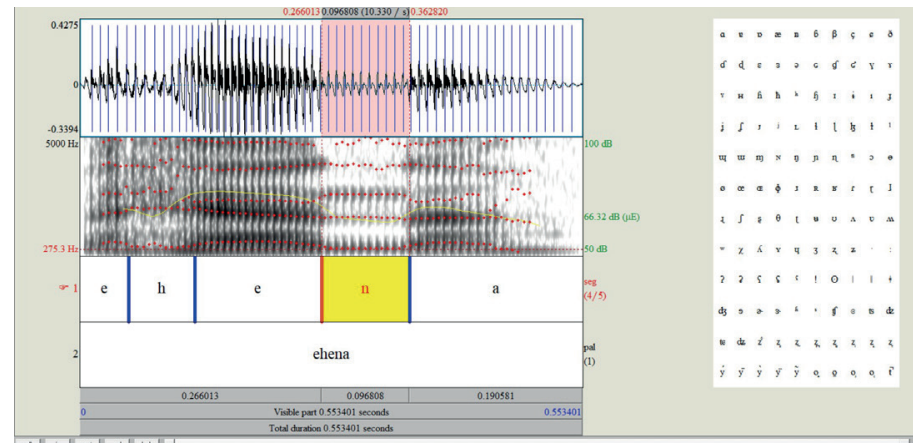

Figura 6. Espectrograma de la palabra [mu.'mø.ri] 'tres'

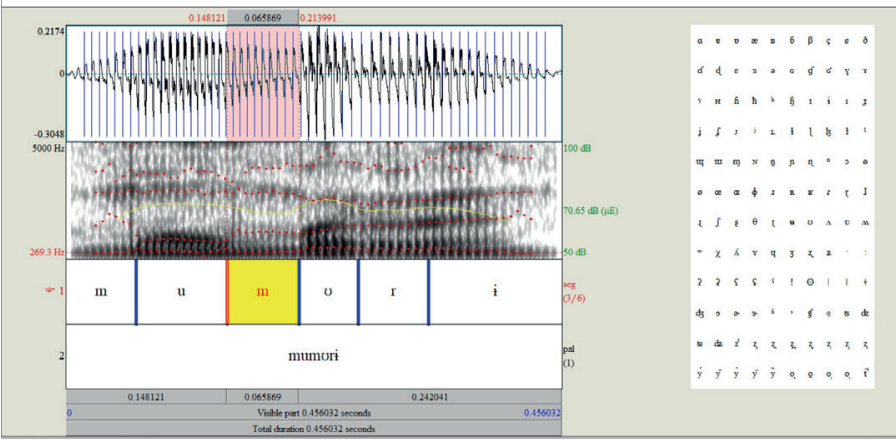

Figura 7. Espectrograma de la palabra [so.næ.'ri.ne] 'oler'

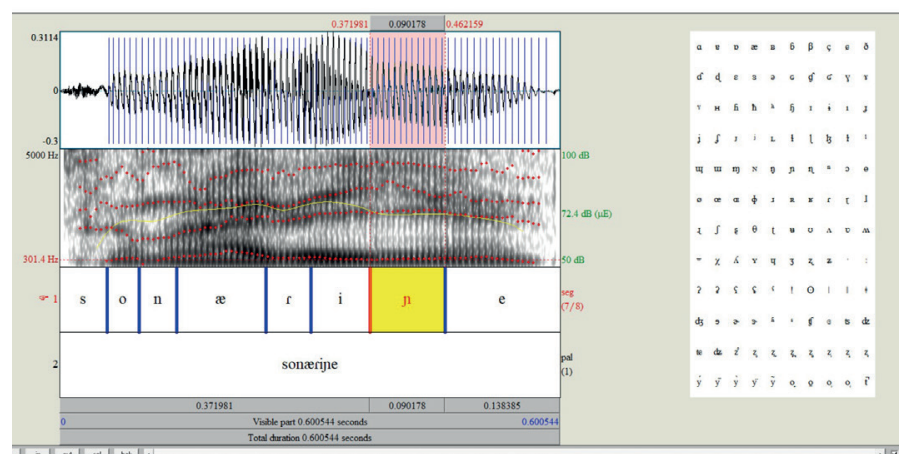

Los valores de la FN y el AF1 se pueden resumir, en promedio, en el cuadro 8 , en el que se indica también el punto de tiempo promedio del segmento en el que se han tomado estos valores.

En cuanto a la duración y la intensidad, véase el cuadro 9 que resume estos valores, incluida la desviación estándar en superíndice y entre paréntesis. 
Guadro 8. Promedios de FN y AF1 en las consonantes nasales

\begin{tabular}{|l|c|c|c|c|}
\hline Consonante & Muestras & $\begin{array}{l}\text { Punto temporal promedio en } \\
\text { el que se tomó la muestra de } \\
\text { FN y AF1 (seg.) (desv. est.) }\end{array}$ & $\begin{array}{l}\text { Promedio de FN } \\
(\mathrm{Hz}) \text { (desv. est.) }\end{array}$ & $\begin{array}{l}\text { Promedio de AF1 } \\
\text { (Hz) (desv. est.) }\end{array}$ \\
\hline$[[\mathrm{m}]$ & 20 & $0.033(0.013)$ & $270(25)$ & $1100(37)$ \\
\hline$[\mathrm{n}]$ & 20 & $0.049(0.016)$ & $288(32)$ & $1200(35)$ \\
\hline$[\mathrm{n}]$ & 15 & $0.045(0.015)$ & $301(38)$ & $2200(39)$ \\
\hline
\end{tabular}

Guadro 9. Promedio de intensidad y duración total de las consonantes nasales del yagua

\begin{tabular}{|l|c|c|c|}
\hline Consonante & Muestras & $\begin{array}{l}\text { Intensidad } \\
\text { (dB) (desv. est.) }\end{array}$ & $\begin{array}{l}\text { Duración } \\
\text { (seg.) (desv. est.) }\end{array}$ \\
\hline$[\mathrm{m}]$ & 20 & $65.136(0.989)$ & $0.066(0.013)$ \\
\hline$[\mathrm{n}]$ & 20 & $66.333(1.254)$ & $0.098(0.016)$ \\
\hline$[\mathrm{n}]$ & 15 & $71.155(1.076)$ & $0.090(0.015)$ \\
\hline
\end{tabular}

\subsection{Consonantes prenasalizadas}

La lengua yagua presenta dos consonantes prenasalizadas: bilabial $\left[{ }^{\mathrm{m} b}\right]$ y alveolar $\left[{ }^{\mathrm{n}} \mathrm{d}\right]$. La principal característica de estos sonidos es, como indica Powlison (2008, p. 32), que aparecen cuando están seguidos de vocales orales en lugar de vocales nasales. En las figuras 8 y 9 se puede observar el espectrograma de estos sonidos, en el que se ve una fase nasal previa a una fase oral característica de las oclusivas. Por su naturaleza articulatoria, esta última siempre es más breve que aquella.

Figura 8. Espectrograma de la palabra [nI.'mbi] 'perro'

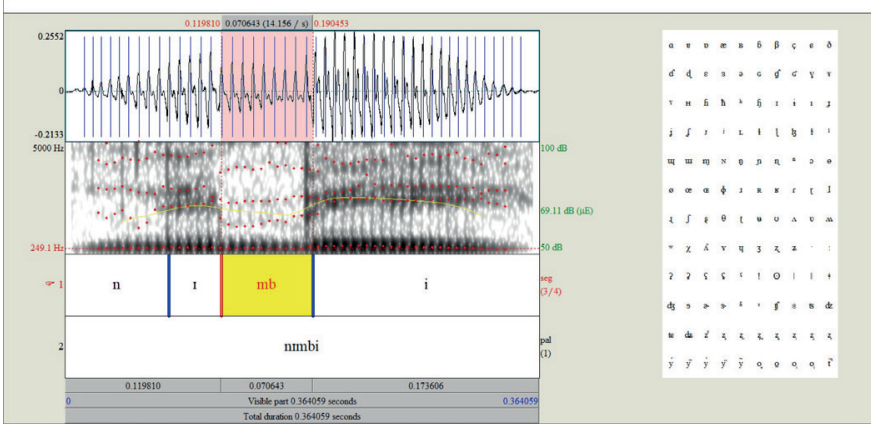

En el cuadro 10 se pueden apreciar los valores de duración obtenidos en la medición de las muestras.

En cuanto a la intensidad y duración de estos sonidos, se obtuvieron los valores expuestos en el cuadro 11 .
Figura 9. Espectrograma de la palabra [sa. $\left.{ }^{\text {nd }} \mathrm{d} \approx\right]$ 'hueso'

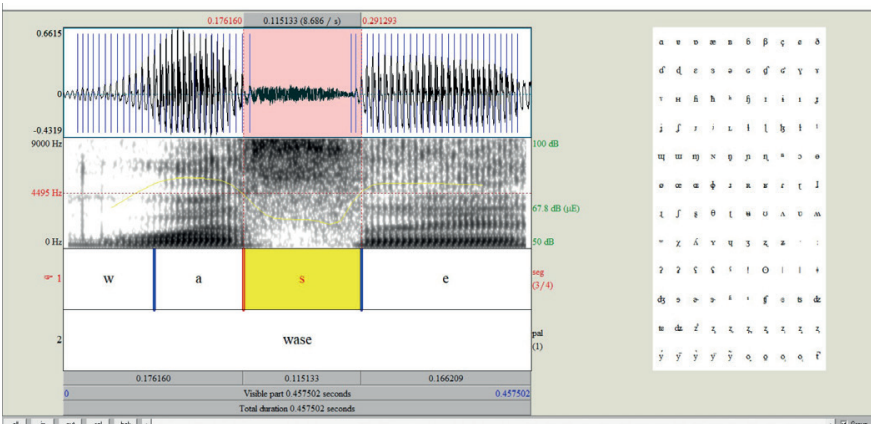

Guadro 10. Duración de la fase de cierre de las prenasalizadas

\begin{tabular}{|l|l|c|}
\hline Consonante & Muestras & Duración (seg.) (desv. est.) \\
\hline$\left[{ }^{m} b\right]$ & 20 & $0.007(0.001)$ \\
\hline$\left[{ }^{n} d\right]$ & 30 & $0.005(0.001)$ \\
\hline
\end{tabular}

Guadro 11. Promedio de intensidad y duración total de las consonantes prenasalizadas

\begin{tabular}{|l|c|l|l|}
\hline Consonante & Muestras & $\begin{array}{l}\text { Intensidad } \\
(\mathrm{dB})(\text { desv. est.) }\end{array}$ & $\begin{array}{l}\text { Duración } \\
(\text { seg.) (desv. est.) }\end{array}$ \\
\hline$\left[{ }^{\mathrm{m}} \mathrm{b}\right]$ & 20 & $69.100(0.930)$ & $0.070(0.014)$ \\
\hline$\left[{ }^{\mathrm{n}} \mathrm{d}\right]$ & 30 & $66.077(0.927)$ & $0.099(0.013)$ \\
\hline
\end{tabular}

\subsection{Consonantes fricativas}

El yagua cuenta con dos consonantes fricativas sordas sibilantes: la alveolar [s] y la alveopalatal [J]. Estos sonidos se caracterizan por presentar ruido aleatorio y alta concentración de energía en las frecuencias superiores a los $2000 \mathrm{~Hz}$. Al respecto, véanse las figuras 10 y 11 .

Como puede observarse, el indicador distintivo de estos sonidos es la aperiodicidad de sus ondas sonoras, que aparecen en el espectrograma como energía dispersa por encima de los $2000 \mathrm{~Hz}$, con una concentración en el intervalo aproximado de 
Figura 10. Espectrograma de la palabra ['wa. se] 'correcto'

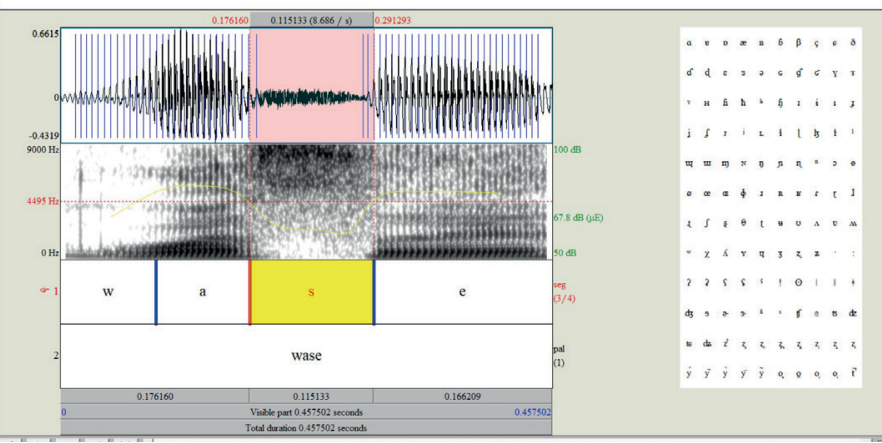

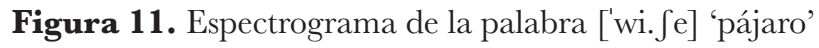

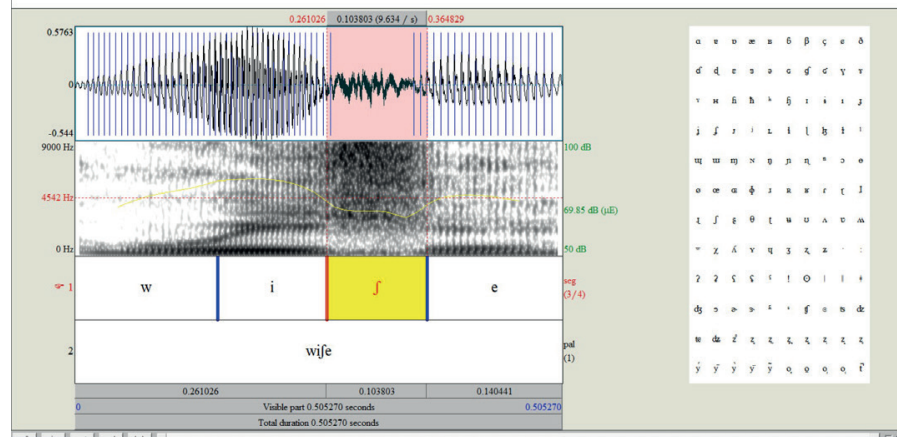

5000 a $9000 \mathrm{~Hz}$ para [s] y a partir de los $2000 \mathrm{~Hz}$ para $\left[\int\right]$.

En cuanto a la duración y la intensidad de los sonidos fricativos sordos, los valores del cuadro 12 fueron obtenidos de palabras en la que los sonidos en cuestión se hallaban al interior de la palabra.

Guadro 12. Promedio de intensidad y duración de las fricativas sibilantes

\subsection{Consonantes africadas}

Se ha registrado palabras en yagua en las que aparece la consonante africada alveopalatal [t $\left.\int\right]$. Según Payne

\begin{tabular}{|l|c|l|l|}
\hline Consonante & Muestras & $\begin{array}{l}\text { Intensidad } \\
(\mathrm{dB}) \text { (desv. est.) }\end{array}$ & $\begin{array}{l}\text { Duración } \\
(\text { seg.) (desv. est.) }\end{array}$ \\
\hline$[\mathrm{s}]$ & 25 & $67.893^{(0.853)}$ & $0.115^{(0.035)}$ \\
\hline$[J]$ & 20 & $69.854^{(0.933)}$ & $0.103^{(0.042)}$ \\
\hline
\end{tabular}

(1985, p. 29), este sonido puede ser un alófono de la fricativa alveolar /s/. No obstante, la presencia de variaciones en la realización de una misma palabra por parte de distintos hablantes yagua parece indicar que podría tratarse, más bien, de una variación de la fricativa alveopalatal $/ \int /$. Al respecto, véanse en figuras 12 y 13 las variantes de la palabra 'pájaro' en yagua.
Como puede observarse, en la figura 12 no hay una fase de oclusión. En cambio, en la palabra de la figura 13 se puede ver que la consonante se

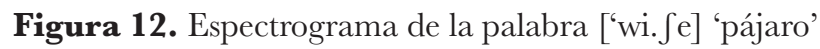
producida por el colaborador MS-V-R (río Napo)

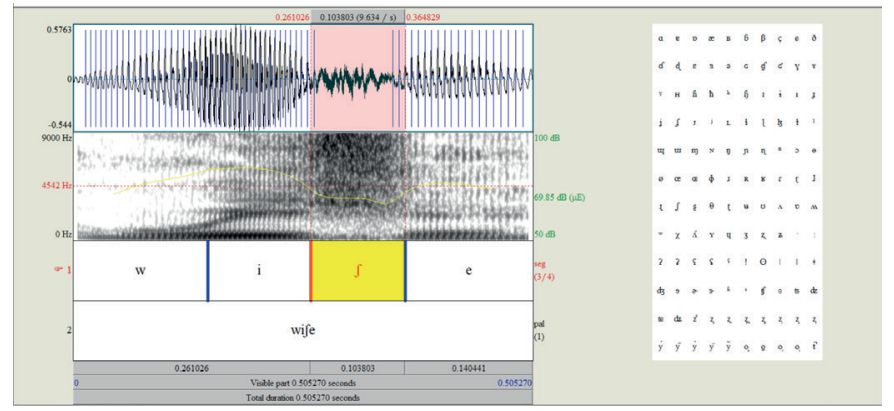

Figura 13. Espectrograma de la palabra ['wi.t $\left.\int e\right]$ 'pájaro' producida por el colaborador CG-V-A (Atacuari)

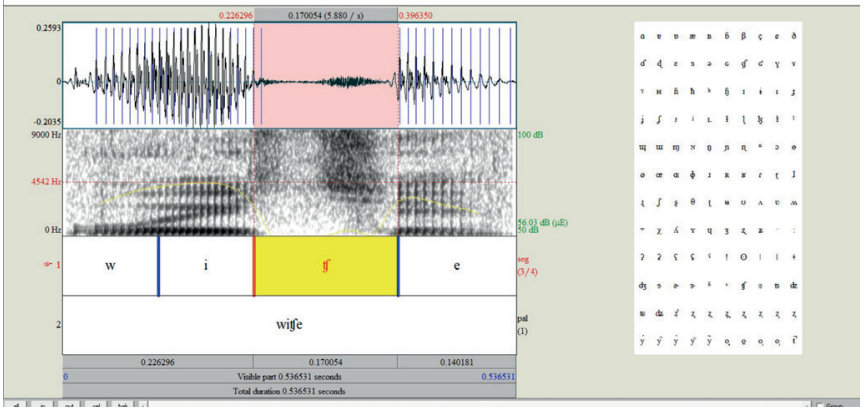

conforma por una fase de oclusión y una de fricación. Acústicamente, estas fases presentan características similares a las de los sonidos oclusivos y fricativos, aunque se debe indicar que la fase de oclusión suele ser más corta que en la de las oclusivas y la fricación dura menos tiempo que en un sonido fricativo simple equivalente. Sin embargo, tal como indica Payne (1985, p. 29), la africada alveopalatal también tiene un estatus fonémico cuyo único correlato fonético es también el sonido africado alveopalatal sordo, aunque no presenta ejemplos del caso. En tal sentido, no se ha podido encontrar casos en que, exclusivamente, un sonido africado no presente su variante fricativa, aunque la muestra sí nos permite afirmar que, en concordancia con Payne (1985), la africada alveopalatal podría ser un fonema y, por lo tanto, su forma fonética no estaría motivada por una regla fonológica, sino que se trataría de una variación dialectal ${ }^{2}$. Al respecto, obsérvese el caso de la palabra 'humo' en las figuras 14, 15 y 16.

En cuanto a la duración, obsérvese los valores de cada fase y la duración total de los sonidos africados en el cuadro 13. 
Figura 14. Espectrograma de la palabra [nu.'wi.t fo] 'humo' producida por el colaborador AG-V-C (Caballococha)

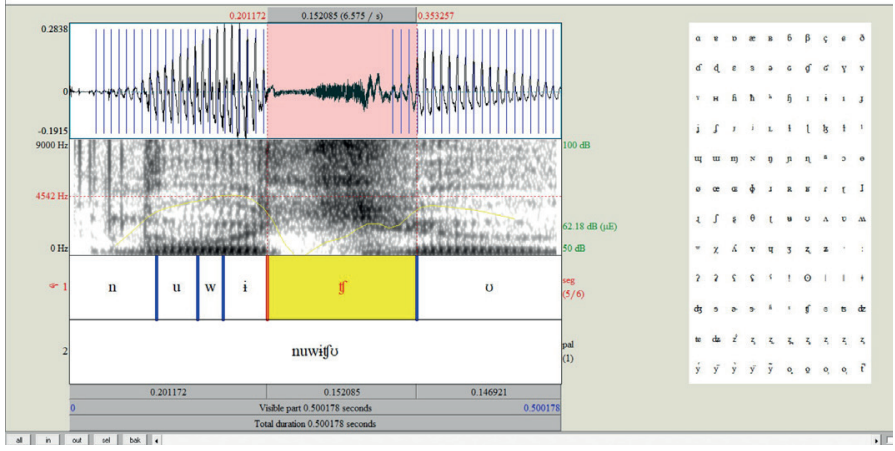

Figura 15. Espectrograma de la palabra [nø.'wa.t $\left.\int \mathrm{I}\right]$ 'humo' producida por el colaborador HQ-V-P (Putumayo)

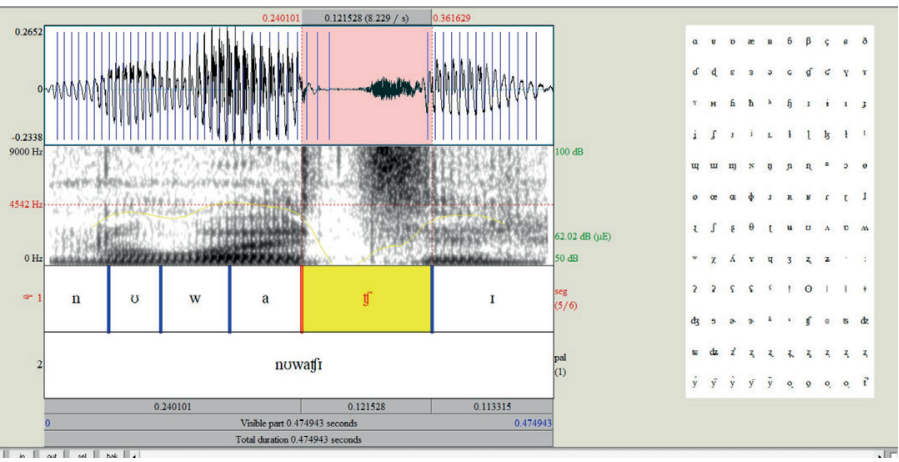

Figura 16. Espectrograma de la palabra [nu.'wa. Ji] 'humo' producida por el colaborador MS-V-R (río Napo)

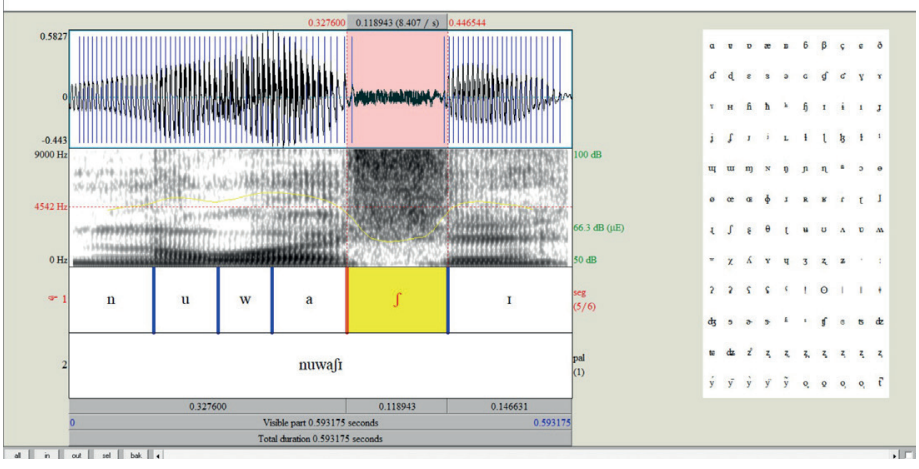

\subsection{Consonantes vibrantes}

Se han registrado tres consonantes vibrantes en el yagua: múltiple $[r]$, simple $[r]$ y retrofleja $[r]$. La vibrante simple, tal como puede observarse en la figura 17, presenta una fase de oclusión y una fase vocálica, semejante a la del español. Además, muestra el mismo patrón de transición de formantes F2 y F3 hacia la vocal consiguiente.

Figura 17. Espectrograma de la palabra [wi.'ti.ro] 'esposa' en la que se puede apreciar el mismo patrón transicional de los formantes de la [r] española

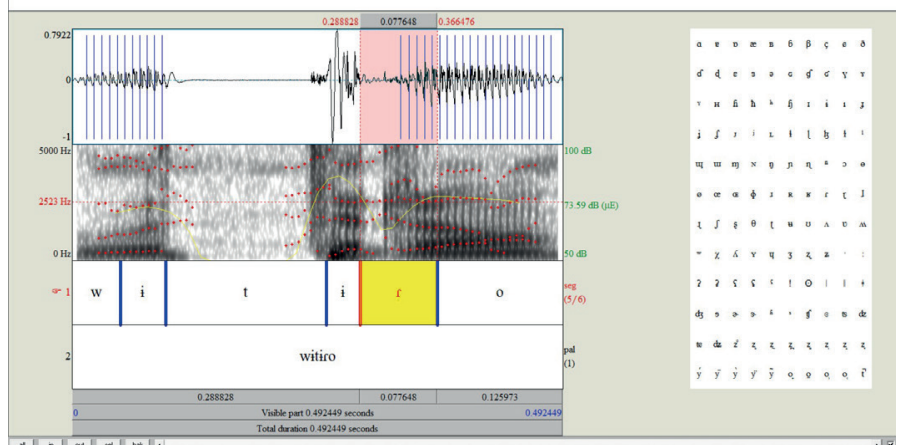

En el cuadro 14 observamos los valores de los formantes de esta consonante.

Guadro14. Valor promedio de los formantes transicionales F2 y F3 de la vibrante simple hacia una vocal posterior $[\mathrm{o}]$

\begin{tabular}{|c|c|c|c|c|c|}
\hline Consonante & Muestras & $\begin{array}{l}\text { Punto temporal } \\
\text { promedio en el } \\
\text { que se tomó la } \\
\text { muestra de F2 } \\
\text { y F3 (seg.) (desv. } \\
\text { est.) } \\
\end{array}$ & $\begin{array}{c}\mathrm{F} 2(\mathrm{~Hz}) \\
\text { (desv. est.) }\end{array}$ & $\begin{array}{c}\mathrm{F3}(\mathrm{Hz}) \\
\text { (desv. est.) }\end{array}$ & $\begin{array}{c}\text { Diferencia } \\
(\mathrm{Hz})\end{array}$ \\
\hline$[\mathrm{r}]$ & 30 & $0.038^{(0.010)}$ & $1600^{(35)}$ & $2600^{(44)}$ & 1000 \\
\hline
\end{tabular}

Por lo que respecta a las vibrantes múltiple y retrofleja parece que ambos sonidos varían según el dialecto. En tal sentido, no se ha evidenciado alternancias entre los dos fonos en ninguno de los hablantes entrevistados. Una pista para suponer ello es el hecho de que Payne (1985) haya registrado un solo fonema vibrante /r/ y su sonido [r] correspondiente. Cabe señalar que la vibrante múltiple solo se ha hallado a inicio de palabra, contexto que supondría un condicionamiento fonológico de fortición para su

Guadro 13. Promedio de intensidad y duración del sonido africado [t $\left.\int\right]$

\begin{tabular}{|c|c|c|c|c|c|}
\hline Consonante & Muestras & $\begin{array}{l}\text { Intensidad (dB) } \\
\text { (desv. est.) }\end{array}$ & $\begin{array}{l}\text { Fase oclusiva } \\
\text { (seg.) (desv. est.) }\end{array}$ & $\begin{array}{l}\text { Fase fricativa } \\
\text { (seg.) (desv. est.) }\end{array}$ & $\begin{array}{l}\text { Promedio de AF1 } \\
(\mathrm{Hz}) \text { (desv. est.) }\end{array}$ \\
\hline$[[\mathrm{t}]$ & 20 & $59.983(1.568)$ & $0.051(0.029)$ & $0.100(0.031)$ & $0.151(0.034)$ \\
\hline
\end{tabular}


realización fonética, fenómeno observable en diversas lenguas, como el español (Lahoz-Bengoechea, 2015, p. 135). Obsérvense las figuras 18 y 19 que muestran dos casos diferentes de la misma palabra.

Figura 18. Espectrograma de la palabra ['ru.ne] 'piojo' (con vibrante múltiple)

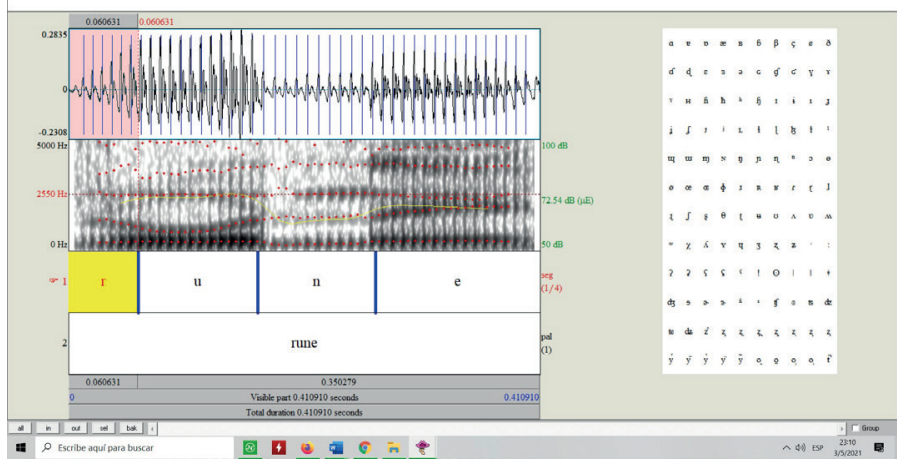

Figura 19. Espectrograma de la palabra [rø.'ne] 'piojo' en la que se puede apreciar que la vibrante presenta un patrón semejante al de los F2 y F3 de [r]

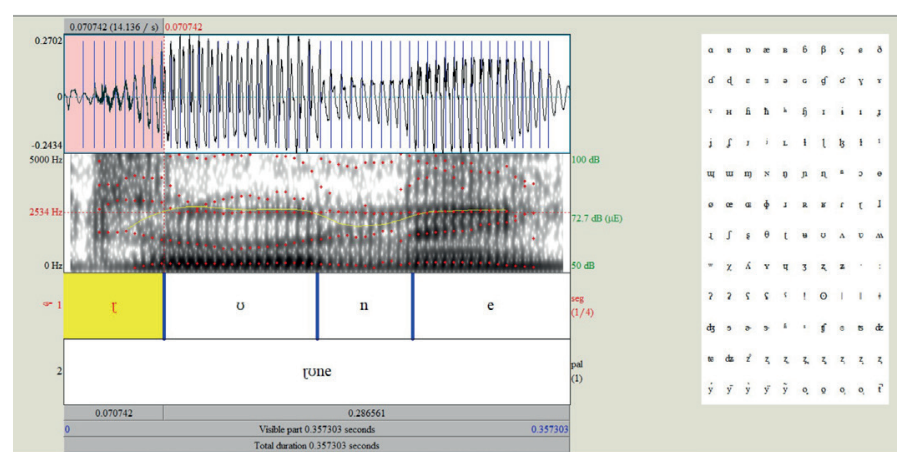

En el caso de la vibrante múltiple, como se ve en la figura 17, se pueden observar las fases de oclusión seguidas de las fases vocálicas características de estos sonidos. En cuanto a la vibrante retrofleja, se aprecia que su aspecto es acústicamente parecido al vibrante simple, pues presenta una sola fase oclusiva y un movimiento proporcional de los valores de su segundo y tercer formante al momento de transición hacia una vocal (Baskt, 2012, p. 34), como se señala en el cuadro 15.

Guadro 15. Valor promedio de los formantes transicionales F2 y F3 de la vibrante retrofleja hacia una vocal posterior [ø]

\begin{tabular}{|l|l|c|c|c|c|}
\hline Consonante & Muestras & $\begin{array}{c}\text { Punto temporal } \\
\text { promedio en el } \\
\text { que se tomó la } \\
\text { muestra de F2 } \\
\text { y F3 (seg.) (desv. } \\
\text { est.) }\end{array}$ & $\begin{array}{c}\text { F2 (Hz) } \\
\text { (desv. est.) }\end{array}$ & $\begin{array}{c}\text { F3 (Hz) } \\
\text { (desv. est.) }\end{array}$ & $\begin{array}{c}\text { Diferencia } \\
(\mathrm{Hz})\end{array}$ \\
\hline$[\mathrm{r}]$ & 15 & $0.036^{(0.009)}$ & $1600^{(36)}$ & $2800^{(47)}$ & 1200 \\
\hline
\end{tabular}

En el cuadro 16, se presentan los valores de tiempo de las tres consonantes vibrantes.

Cuadro 16. Promedio de intensidad y duración de las

\begin{tabular}{|c|c|c|c|}
\hline Consonante & Muestras & $\begin{array}{l}\text { Intensidad } \\
\text { (dB) (desv. est.) }\end{array}$ & $\begin{array}{l}\text { Duración } \\
\text { (seg.) (desv. est.) }\end{array}$ \\
\hline$[\mathrm{r}]$ & 30 & $73.856^{(0.931)}$ & $0.077^{(0.010)}$ \\
\hline$[r]$ & 10 & $72.573^{(0.790)}$ & $0.063^{(0.022)}$ \\
\hline [r] & 15 & $72.776^{(0.811)}$ & $0.071^{(0.009)}$ \\
\hline
\end{tabular}

\subsection{Consonantes aproximantes}

Se han registrado cuatro sonidos aproximantes en yagua: palatal [j], labiovelar [w], bilabial [ $\beta$ ] y aspirado [h]. Para la aproximante palatal [j], se observa un F1 por debajo de los $500 \mathrm{~Hz}$ y un F2 alrededor de los $2000 \mathrm{~Hz}$, semejante al de la vocal [i]. Por su parte, la aproximante labiovelar $[\mathrm{w}]$ presenta un F1 también inferior a los $500 \mathrm{~Hz}$, pero un F2 alrededor de los $1000 \mathrm{~Hz}$ similar al de la vocal [u].

Cuadro 17. Valor promedio de los formantes F1 y F2 de las aproximantes [j] y [w]

\begin{tabular}{|l|l|l|l|}
\hline Consonante & Muestras & $\mathrm{F} 1(\mathrm{~Hz})$ & $\mathrm{F} 2(\mathrm{~Hz})$ \\
\hline$[\mathrm{j}]$ & 20 & $370^{(32)}$ & $2250^{(201)}$ \\
\hline$[\mathrm{w}]$ & 20 & $360^{(25)}$ & $950^{(78)}$ \\
\hline
\end{tabular}

En ambos casos, la intensidad de energía es menor a la de una vocal, tal como se puede apreciar en las manchas blancas que se encuentran entre sus formantes en las figuras 20 y 21 .

Figura 20. Espectrograma de la palabra [ja.ru.'we] 'partir' ('romper en dos')

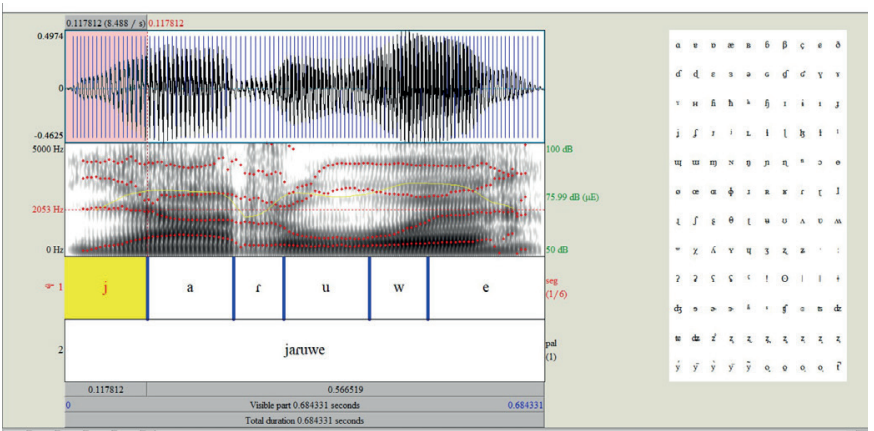


Figura 21. Espectrograma de la palabra [a.wi.'ne] 'animal'

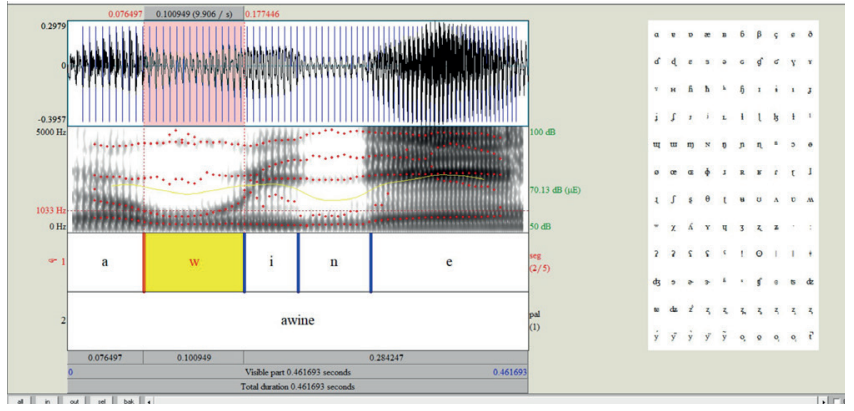

En el siguiente cuadro, se observan los promedios de intensidad y duración de estos sonidos.

Guadro 18. Duración de las aproximantes [j] y [w]

\begin{tabular}{|l|c|l|l|}
\hline Consonante & Muestras & $\begin{array}{l}\text { Intensidad } \\
(\mathrm{dB})^{(\text {desv. est.) }}\end{array}$ & $\begin{array}{l}\text { Duración } \\
\text { (seg.) }^{\text {(desv. est.) }}\end{array}$ \\
\hline$[\mathrm{j}]$ & 20 & $75.692^{(0.884)}$ & $0.115^{(0.020)}$ \\
\hline$[\mathrm{w}]$ & 20 & $70.148^{(0.969)}$ & $0.101^{(0.014)}$ \\
\hline
\end{tabular}

En el caso de $[\beta]$, se observa la periodicidad de este sonido en posición intervocálica, lo que se puede reconocer como la continuidad de las estrías de los formantes de las vocales que lo rodean, aunque con menor intensidad, pues se observan manchas blancas entre las estrías. Esto demuestra que no hay turbulencia como en los sonidos fricativos.

Figura 22. Espectrograma de la palabra [a.'ßקe.ti.ro] 'esposa'

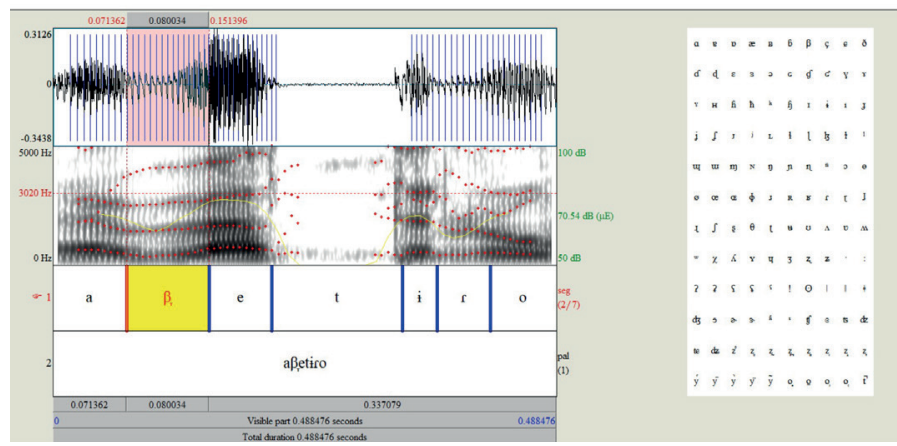

Cuadro 19. Promedio de intensidad y duración de la aproximante $[\beta]$

\begin{tabular}{|l|c|l|c|}
\hline Consonante & Muestras & $\begin{array}{l}\text { Intensidad } \\
(\mathrm{dB})^{(\text {desv. est.) }}\end{array}$ & $\begin{array}{l}\text { Duración } \\
\text { (seg.) }^{\text {(desv. est.) }}\end{array}$ \\
\hline$[\beta]$ & 10 & $71.008^{(0.721)}$ & $0.079^{(0.009)}$ \\
\hline
\end{tabular}

En cuanto a $[\mathrm{h}]$, aunque suele categorizársela como fricativa, cabe recordar que es mejor considerarla como un sonido aproximante glotal que no presenta articulación ni en la cavidad oral ni en la nasal (Crystal, 2008, p. 32; Ladefoged y Maddieson, 1996, p. 326). Obsérvese el espectrograma de una muestra de este sonido en la siguiente figura.

Figura 23. Espectrograma de la palabra ['hi.na] 'pesado'

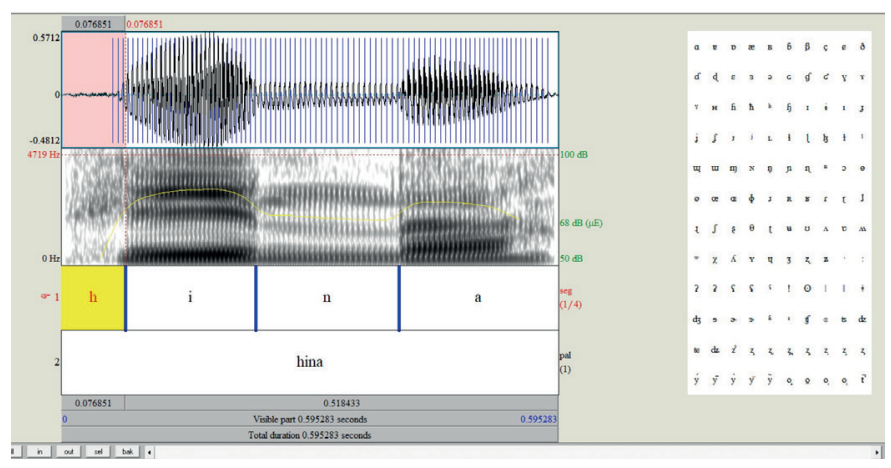

En el cuadro 20, se puede observar el promedio de intensidad y duración de este sonido sobre la base de las muestras.

Cuadro 20. Duración de la aproximante [h]

\begin{tabular}{|l|c|l|l|}
\hline Consonante & Muestras & $\begin{array}{l}\text { Intensidad } \\
(\mathrm{dB})^{(\text {desv. est.) }}\end{array}$ & $\begin{array}{l}\text { Duración } \\
\text { (seg.) }^{(\text {desv. est.) }}\end{array}$ \\
\hline$[\mathrm{h}]$ & 25 & $67.964^{(0.818)}$ & $0.077^{(0.017)}$ \\
\hline
\end{tabular}

\subsection{Articulaciones secundarias}

Se ha podido identificar la aproximante velar [w] como una articulación secundaria adyacente a las consonantes bilabiales. En efecto, tal como indica Payne (1985, p. 29), esta articulación se produciría en el alófono $\left[\mathrm{p}^{\mathrm{w}}\right] \mathrm{de} / \mathrm{p} / \mathrm{y}\left[\mathrm{m}^{\mathrm{w}}\right] \mathrm{de} / \mathrm{m} /$. En concordancia con ello, Powlison (2008, p. 31) señala que este sonido aparece cuando una consonante bilabial se encuentra ante la vocal [i]. Cabe indicar que, en lenguas como el shipibo, también se presenta esta regla fonológica, la cual "puede ocurrir tanto en sílabas iniciales como no iniciales de una palabra, así como también en sílabas acentuadas y no-acentuadas" (Elías-Ulloa, 2011, p. 77).

Este fenómeno es conocido como labialización, aunque a veces se le denomina también labiovelarización; no obstante, autores como Ladefoged prefieren usar el término labialización (Ladefoged y Maddieson, 
1996, p. 356), tal como coincide también ElíasUlloa (2011). En tal sentido, no se debe confundir con lo que Ladefoged denomina labialización simple, que se diferencia del anterior fenómeno en que solo compromete el redondeamiento de labios, es decir, sin el alzamiento del posdorso de la lengua (Ladefoged y Maddieson, 1996, p. 356).

De acuerdo con estas observaciones y con el análisis de las muestras, se puede comprobar que, en el caso del yagua, esta articulación secundaria se origina a partir del condicionamiento fonológico de la vocal [i]. Obsérvese, a continuación, los espectrogramas de palabras en las que se evidencian los casos de $\left[\mathrm{m}^{\mathrm{w}}\right]$ y $\left[\mathrm{p}^{\mathrm{w}}\right]$.

Figura 24. Espectrograma de la palabra ['mi $\mathrm{i}]$ 'suciedad'

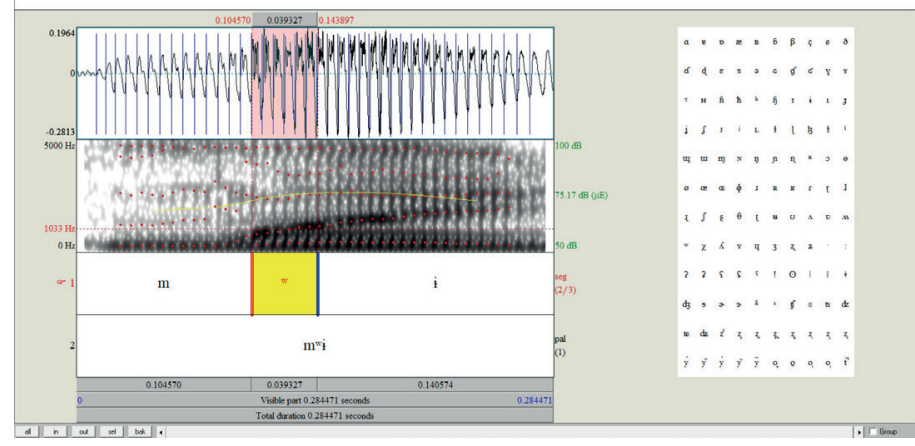

Figura 25. Espectrograma de la palabra [ja.cひ.'p $\mathrm{p}^{\mathrm{w} i}$ ] 'caminar'

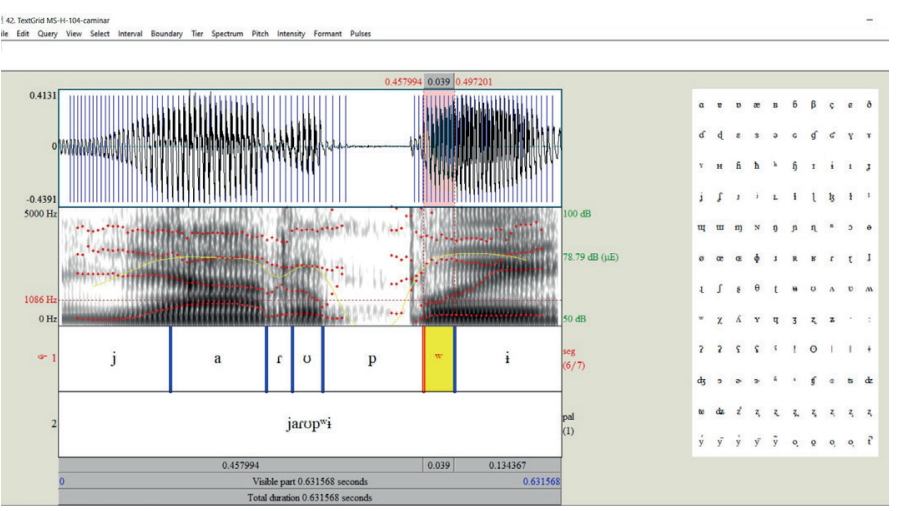

En cuanto a las propiedades acústicas de [" $\left.{ }^{\mathrm{w}}\right]$, estas son similares a las de $[\mathrm{w}]$, aunque se observa una ligera elevación de los F1 y F2 en el caso de la articulación secundaria. No obstante, la diferencia notable se halla en la duración de ambos sonidos, pues $[\mathrm{w}]$, al tratarse de una consonante independiente, presenta una duración mayor. En el cuadro 21, se pueden apreciar estos valores.
Guadro 21. Comparación de los valores de la aproximante $[\mathrm{w}]$, la articulación secundaria $\left.{ }^{[\mathrm{w}}\right]$ y la vocal alta central no redondeada [i]

\begin{tabular}{|l|l|l|l|l|l|}
\hline Consonante & Muestras & $\mathrm{F} 1(\mathrm{~Hz})$ & $\begin{array}{c}\mathrm{F} 2(\mathrm{~Hz}) \\
\text { (desv. est.) }\end{array}$ & $\begin{array}{l}\text { Intensidad (dB) } \\
\text { (desv. est.) }\end{array}$ & $\begin{array}{l}\text { Duración } \\
\text { (seg.) } \\
\text { (desv. est.) }\end{array}$ \\
\hline$[\mathrm{w}]$ & 18 & $380^{(11)}$ & $970^{(46)}$ & $79.338^{(0.905)}$ & $0.039^{(0.003)}$ \\
\hline$[\mathrm{w}]$ & 20 & $360^{(25)}$ & $950^{(78)}$ & $70.148^{(0.836)}$ & $0.101^{(0.014)}$ \\
\hline
\end{tabular}

\section{Discusión}

En la mayoría de los casos, la descripción acústica realizada en esta investigación ha coincidido con las propuestas articulatorias de los autores. En los que no ha sido así, es decir, con las vibrantes y la africada [ț], se debe tomar en cuenta el factor de la variación y el cambio lingüístico, los cuales podrían ser la razón de las diferencias. En tal sentido, se ha podido observar la presencia de tres tipos de vibrantes: múltiple [r], simple $[\mathrm{r}]$ y retrofleja $[\mathrm{r}]$.

Cabe señalar que la vibrante múltiple solo se ha hallado a inicio de palabra, lo que supondría un condicionamiento fonológico de fortición para su realización fonética, que podría responder a procesos de castellanización que se pueden proponer como investigaciones posteriores. Asimismo, se ha podido comprobar que la aparición de la segunda articulación [ ${ }^{\mathrm{w}}$ ] solo ocurre cuando los sonidos [p] y $[\mathrm{m}]$ se encuentran ante una vocal central [i]. Del mismo modo, se ha podido comprobar que los sonidos prenasalizados $\left[{ }^{\mathrm{m}} \mathrm{b}\right]$ y $\left[{ }^{\mathrm{n}} \mathrm{d}\right]$ aparecen ante vocales orales, tal como lo describe Powlison (2008).

En cuanto a la variación fonética, se puede afirmar que la influencia creciente del español podría explicar, por ejemplo, el hecho de que existan sonidos vibrantes múltiples y simples que no habían sido reportados por investigadores anteriores. Aparte de ello, debe tomarse en cuenta la influencia de la lengua hispana sobre la lengua yagua en la medida en que las nuevas generaciones ya no son monolingües, lo cual la ha puesto en condición de amenazada.

\section{Conclusión}

Se han descrito las propiedades acústicas de 20 sonidos consonánticos en yagua: $[\mathrm{p}],\left[\mathrm{p}^{\mathrm{w}}\right],[\mathrm{t}],[\mathrm{k}]$, 
$[\mathrm{m}],\left[\mathrm{m}^{\mathrm{w}}\right],\left[{ }^{\mathrm{m} b}\right],[\mathrm{n}],[\mathrm{p}],\left[{ }^{\mathrm{n}} \mathrm{d}\right],[\mathrm{t} 丁],[\mathrm{s}],\left[\int\right],[\mathrm{r}],[\mathrm{r}]$, $[\mathrm{c}],[\mathrm{w}],[\beta],[j]$ y $[\mathrm{h}]$. Estos quedarían clasificados articulatoriamente como se muestra en el cuadro 22.

Guadro 22. Inventario de los sonidos consonánticos del yagua

\begin{tabular}{|l|l|c|l|l|l|}
\hline & Labial & Alveolar & Palatal & Velar & Glotal \\
\hline Oclusiva & $\begin{array}{l}{[\mathrm{p}]} \\
{\left[\mathrm{p}^{\mathrm{w}}\right]}\end{array}$ & {$[\mathrm{t}]$} & & & \\
\hline & {$[\mathrm{m}]$} & {$[\mathrm{n}]$} & & & \\
\hline Nasal & {$\left[\mathrm{m}^{\mathrm{w}}\right]$} & {$[\mathrm{n}]$} & & & \\
& {$\left[\mathrm{m}^{\mathrm{b}}\right]$} & {$[\mathrm{n} d]$} & & & \\
\hline Africada & & & {$[\mathrm{t}]$} & & \\
\hline Fricativa & & {$[\mathrm{s}]$} & & & \\
\hline Vibrante & & {$[\mathrm{r}]$} & & & \\
& & {$[\mathrm{r}]$} & & & \\
& & {$[\mathrm{r}]$} & & & \\
\hline Aproximante & {$[\mathrm{w}]$} & & & & \\
& {$[\beta]$} & & {$[\mathrm{j}]$} & & {$[\mathrm{h}]$} \\
\hline
\end{tabular}

\section{Anexo I}

Lista de 200 palabras de Swadesh

\begin{tabular}{|lll|}
\hline & Palabra en inglés & Palabra en español \\
\hline 1 & animal & animal \\
\hline 2 & dog & perro \\
\hline 3 & bird & pájaro \\
\hline 4 & snake & culebra \\
\hline 5 & fish & pez, pescado \\
\hline 6 & louse & piojo \\
\hline 7 & worm & lombriz \\
\hline 8 & grass & pasto \\
\hline 9 & tree & árbol \\
\hline 10 & name & nombre \\
\hline 11 & father & padre \\
\hline 12 & mother & madre \\
\hline 13 & husband & esposo \\
\hline 14 & wife & esposa \\
\hline 15 & man & hombre \\
\hline 16 & woman & mujer \\
\hline & & \\
\hline
\end{tabular}

\section{Anexo I}

Lista de 200 palabras de Swadesh

\begin{tabular}{|c|c|c|}
\hline & Palabra en inglés & Palabra en español \\
\hline 17 & child & niño \\
\hline 18 & person & persona \\
\hline 19 & head & cabeza \\
\hline 20 & ear & oreja \\
\hline 21 & eye & ojo \\
\hline 22 & nose & nariz \\
\hline 23 & mouth & boca \\
\hline 24 & tongue & lengua \\
\hline 25 & tooth & diente \\
\hline 26 & neck & cuello \\
\hline 27 & belly & barriga \\
\hline 28 & back & espalda \\
\hline 29 & tail & rabo, cola \\
\hline 30 & leg & pierna \\
\hline 31 & foot & pie \\
\hline 32 & wing & ala \\
\hline 33 & hand & mano \\
\hline 34 & heart & corazón \\
\hline 35 & guts & tripas, intestinos \\
\hline 36 & liver & hígado \\
\hline 37 & bone & hueso \\
\hline 38 & meat & carne \\
\hline 39 & fat & grasa \\
\hline 40 & skin & piel \\
\hline 41 & hair & cabello (o pelo) \\
\hline 42 & feather & pluma \\
\hline 43 & blood & sangre \\
\hline 44 & root & raíz \\
\hline 45 & bark & corteza \\
\hline 46 & leave & hoja \\
\hline 47 & flower & flor \\
\hline 48 & fruit & fruta \\
\hline 49 & seed & semilla \\
\hline 50 & stick & palo \\
\hline 51 & ashes & ceniza \\
\hline 52 & mountain & montaña \\
\hline 53 & forest & bosque \\
\hline
\end{tabular}




\begin{tabular}{|c|c|c|c|c|c|}
\hline 54 & river & río & 94 & to fall & caer-(se) \\
\hline 55 & lake & lago & 95 & to breathe & respirar \\
\hline 56 & sea & mar & 96 & to blow & soplar \\
\hline 57 & water & agua & 97 & to sleep & dormir \\
\hline 58 & ice & hielo & 98 & to lean & echar-(se) \\
\hline 59 & fire & fuego & 99 & to sit & sentar-(se) \\
\hline 60 & smoke & humo & 100 & to stop & parar-(se) \\
\hline 61 & earth & tierra & 101 & to float & flotar, rebalsar \\
\hline 62 & dust & polvo & 102 & to flow & fluir \\
\hline 63 & sand & arena & 103 & to come & venir \\
\hline 64 & stone & piedra & 104 & to walk & caminar, andar \\
\hline 65 & road & carretera (camino, trocha) & 105 & to fly & volar \\
\hline 66 & egg & huevo & 106 & to swim & nadar \\
\hline 67 & rain & Iluvia & 107 & to turn & voltear, girar \\
\hline 68 & snow & nieve & 108 & to play & jugar \\
\hline 69 & fog & neblina & 109 & to see & ver \\
\hline 70 & sky & cielo & 110 & to smell & oler \\
\hline 71 & cloud & nube & 111 & to listen & oír \\
\hline 72 & wind & viento & 112 & to know & saber \\
\hline 73 & sun & sol & 113 & to think & pensar \\
\hline 74 & star & estrella & 114 & to fear & temer \\
\hline 75 & day & día & 115 & to count & contar \\
\hline 76 & night & noche & 116 & to say & decir \\
\hline 77 & year & año & 117 & to sing & cantar \\
\hline 78 & rope & cuerda & 118 & to laugh & reír \\
\hline 79 & salt & sal & 119 & to eat & comer \\
\hline 80 & I & yo & 120 & to drink & beber, tomar \\
\hline 81 & You & tú, usted & 121 & to suck & chupar \\
\hline 82 & $\mathrm{He}$ & él & 122 & to bite & morder \\
\hline 83 & They & ellos & 123 & to spit & escupir \\
\hline 84 & We & nosotros & 124 & to vomit & vomitar \\
\hline 85 & You & ustedes, vosotros & 125 & to scratch & rascar \\
\hline 86 & who? & ¿quién? & 126 & to hunt & cazar \\
\hline 87 & what? & ¿qué? & 127 & to burn & quemar \\
\hline 88 & this & este & 128 & to sew & coser \\
\hline 89 & that & ese, aquel & 129 & to tie & atar, amarrar \\
\hline 90 & to live & vivir & 130 & to pull & jalar \\
\hline 91 & to die & morir & 131 & to throw & tirar, lanzar \\
\hline 92 & freeze & congelar, helarse & 132 & to push & empujar \\
\hline 93 & to swell & hinchar & 133 & to squeeze & apretar \\
\hline
\end{tabular}




\begin{tabular}{|c|c|c|}
\hline 134 & to dig & cavar, escarbar \\
\hline 135 & to wash & lavar \\
\hline 136 & to clean & limpiar, enjugar \\
\hline 137 & to rub & sobar, frotar \\
\hline 138 & to give & dar \\
\hline 139 & to grab & agarrar, sostener \\
\hline 140 & to cut & cortar \\
\hline 141 & to split & partir \\
\hline 142 & to fight & pelear, luchar \\
\hline 143 & to hit & golpear \\
\hline 144 & to stab & perforar \\
\hline 145 & to kill & matar \\
\hline 146 & smooth & liso \\
\hline 147 & hot & caliente \\
\hline 148 & cold & frío \\
\hline 149 & sharp & filudo \\
\hline 150 & dull & desafilado \\
\hline 151 & rotten & podrido \\
\hline 152 & straight & recto, derecho \\
\hline 153 & dirty & sucio \\
\hline 154 & heavy & pesado \\
\hline 155 & wet & mojado \\
\hline 156 & dry & seco \\
\hline 157 & black & negro \\
\hline 158 & white & blanco \\
\hline 159 & red & rojo \\
\hline 160 & yellow & amarillo \\
\hline 161 & green & verde \\
\hline 162 & new & nuevo \\
\hline 163 & old & viejo \\
\hline 164 & good & bueno \\
\hline 165 & bad & malo \\
\hline 166 & here & aquí, acá \\
\hline 167 & there & ahí, allí, allá \\
\hline 168 & close & cerca \\
\hline 169 & far & lejos \\
\hline 170 & right & derecha \\
\hline 171 & left & izquierda \\
\hline 172 & big & grande \\
\hline 173 & wide & ancho \\
\hline
\end{tabular}

\begin{tabular}{|c|c|c|}
\hline 174 & thick & grueso \\
\hline 175 & small & pequeño \\
\hline 176 & thin & delgado \\
\hline 177 & narrow & angosto, estrecho \\
\hline 178 & long & largo \\
\hline 179 & short & corto, bajo \\
\hline 180 & one & uno \\
\hline 181 & two & dos \\
\hline 182 & three & tres \\
\hline 183 & four & cuatro \\
\hline 184 & five & cinco \\
\hline 185 & many & muchos \\
\hline 186 & few & pocos \\
\hline 187 & all & todos \\
\hline 188 & some & algunos \\
\hline 189 & other & otro \\
\hline 190 & where? & ¿dónde? \\
\hline 191 & when? & ¿cuándo? \\
\hline 192 & how? & ¿cómo? \\
\hline 193 & in & en, adentro \\
\hline 194 & right & correcto \\
\hline 195 & no & no \\
\hline 196 & and & $\mathrm{y}$ \\
\hline 197 & because & porque \\
\hline 198 & if & si \\
\hline 199 & at & en \\
\hline 200 & with & con \\
\hline
\end{tabular}

Adaptado de Aguilar-Porras, 2013, pp. 125-129.

\section{Lista de abreviaturas}

AF1 Antiformante 1

dB Decibelio

desv. est. Desviación estándar

F1 Formante 1

F2 Formante 2

F3 Formante 3

FN Formante Nasal

$\mathrm{Hz} \quad$ Hertzio

seg. segundos

VOT Voice Onset Time (tiempo de inicio de voz) 


\section{Notas}

1 Se ha optado por emplear los símbolos del Alfabeto Fonético Internacional en lugar de utilizar los símbolos que utiliza Payne (1985).

2 En comunicación personal, algunos colaboradores bilingües (yagua-castellano) manifestaban que el sonido en cuestión era propio de "algunos dialectos" del yagua.

\section{Referencias bibliográficas}

Aguilar-Porras, E. (2013). La lexicografía y la lista Swadesh: ¿Un método efectivo para establecer un vínculo genealógico entre lenguas? Repertorio Americano, 23. Heredia: UNA. Recuperado de https://www.revistas.una.ac.cr/index.php/repertorio/article/view/7989/9021

Ashby, P. (2011). Understanding Phonetics. Hodder Education.

Baskt, S. (2012). Rhotics and Retroflexes in Indic and Dravidian. University of Cambridge.

Chaumeil, J. P. (1987). Nihamwo: los yagua del nor-oriente peruano. Centro Amazónico de Antropología y Aplicación Práctica.

Chaumeil, J. P. (1994). Los yagua. Guía etnográfica de la Alta Amazonía, vol. I: Mai huna, Yagua, Ticuna. Instituto Francés de Estudios Andinos.

Crystal, D. (2008). A Dictionary of Linguistics and Phonetics. Blackwell.

Elías-Ulloa, J. (2011). Una documentación acústica de la lengua shipibo-conibo (Pano) (Con un bosquejo fonológico). Pontificia Universidad Católica del Perú.

Jiménez, J. (2018). Los sonidos de la lengua arabela: un bosquejo fonológico. Universidad Nacional Mayor de San Marcos.

Johnson, K. (2003). Acoustic and Auditory Phonetics. Blackwell.

Ladefoged, P. y Johnson, K. (2010). A Course in Phonetics. Boston: Wadsworth.

Ladefoged, P. y Maddieson, I. (1996). The sounds of the World's Languages. Blackwell.

Lahoz-Bengoechea, J. (2015). Fonética y fonología de los fenómenos de refuerzo consonántico en el seno de unidades léxicas en español. Universidad Complutense de Madrid.

Ministerio de Educación. (2013). Documento Nacional de Lenguas Originarias del Perú. MineduDirección General de Educación Intercultural Bilingüe y Rural.

Payne, D. (1985). Aspects of the Grammar of Yagua: a Typological Perspective. (Tesis de Doctorado). Universidad de California, Los Ángeles, Estados Unidos de América. https://linguistics.ucla. edu/images/stories/Payne.1985.pdf

Peña, J. (2009). A Historical Reconstruction of the Peba-yaguan Linguistic Family. University of Oregon.

Powlison, P. (1962). Palatalization portmanteaus in Yagua (Peba-Yaguan). WORD, 18(1-3), 280-299. https://doi.org/10.1080/00437956.1962.11659779

Powlison, P. (2008 [1995]). Diccionario yagua-castellano (2. ${ }^{\text {a }}$ ed.). Ministerio de Educación, Instituto Lingüístico de Verano. https://bit.ly/2YjzeLn. 